\title{
Cyclodextrins in polymer synthesis: Dimethyl- $\beta$-cyclodextrin as thermosensitive retardant for the radical polymerization of $N$-methacryloyl-L-tyrosine derivatives in water
}

\author{
Monir Tabatabai, Helmut Ritter*, Monika Schmelzer
}

Heinrich-Heine University of Düsseldorf, Institute of Organic and Macromolecular

Chemistry II, Universitätsstr. 1, 40225 Düsseldorf, Germany; Fax +49-211-81 14788;

h.ritter@uni-duesseldorf.de

(Received: July 22, 2002; published: September 13, 2002)

\begin{abstract}
The synthesis and characterization of $N$-methacryloyl-L-tyrosine methyl ester (3a) and ethyl ester (3b), and their acetyl derivatives $O$-acetyl- $N$-methacryloyl-L-tyrosine methyl ester (4a) and ethyl ester (4b) are described. Monomers 3 and 4 were complexed with RAMEB (randomly methylated $ß$-cyclodextrin) yielding water soluble host-guest complexes $5 \mathbf{a}$-d. The radical polymerization of monomers $\mathbf{3}$ and $\mathbf{4}$ was investigated in the presence as well as in the absence of RAMEB in aqueous medium at room temperature and also at $60^{\circ} \mathrm{C}$. It is shown that the polymerization tendency of complexes $\mathbf{5 a - d}$ at room temperature is lower, leading to polymers of higher molecular weight, compared to the free monomers 3 and 4. Furthermore, the polymerization of monomers 3 and 4 was carried out in homogenous organic solution using 2,2'-azoisobutyronitrile as initiator, and the results are discussed.
\end{abstract}

\section{Introduction}

Cyclodextrins (CDs) and their chemically modified derivatives attract rapidly increasing attention in a large number of applications, e.g., food chemistry as well as pharmaceutical and cosmetic industries, since they are commercially available on a large scale [1-4]. CDs are obtained by modification of starch via degradation of helical polysaccharides into ring shaped molecules. They are cyclic oligomers consisting of $6(\alpha), 7(\beta)$ and $8(\gamma)$ glucopyranose units of 1,4-linked glucose. Due to their polar hydrophilic outer shell and relatively hydrophobic cavity, they are able to build up host-guest complexes by inclusion of suitable hydrophobic molecules. The formation of an inclusion complex leads to a significant change of solubility and reactivity of the guest molecule. Thus, water insoluble molecules may become completely water soluble simply by mixing with an aqueous solution of cyclodextrins or their derivatives. Hydrogen bonds or hydrophobic interactions are responsible for the stability of the host-guest complexes.

In polymer chemistry, cyclodextrins were used to prepare different kinds of mainchain and side-chain polyrotaxanes [5-8]. We have recently investigated the radical polymerization of water soluble complexes of hydrophobic monomers (e.g., methacrylic derivatives) with methylated cyclodextrin in water [9-19]. In all cases, relatively high polymerization rates and high molecular weights were observed in the presence 
of cyclodextrin derivatives. Up to now, the preparation of chiral phenolic monomers bearing a polymerizable methacrylic function and their polymerization in water has not yet been published. The present paper describes the preparation of 1:1 RAMEB (randomly methylated $B$-cyclodextrin) complexes of $L$-tyrosine derivatives and their free radical initiated polymerization in water.

\section{Results and discussion}

\section{Monomer synthesis and complexation}

$N$-Methacryloyl-L-tyrosine esters $\mathbf{3 a}$ and $\mathbf{3} \mathbf{b}$ were prepared by reacting trimethylsilylprotected $L$-tyrosine esters $\mathbf{1 a}$ and $\mathbf{1 b}$ with methacryloyl chloride $\mathbf{2}$. The silyl groups were simply removed by hydrolysis. The synthesis of acetyl derivatives $\mathbf{4 a}$ and $\mathbf{4 b}$ was carried out by the reaction of $\mathbf{3 a}$ or $\mathbf{3 b}$ with acetyl chloride in the presence of triethyl amine (Scheme 1). The monomers were characterized by ${ }^{1} \mathrm{H} N M R, I R$ spectroscopy and X-ray analysis (Fig. 1).

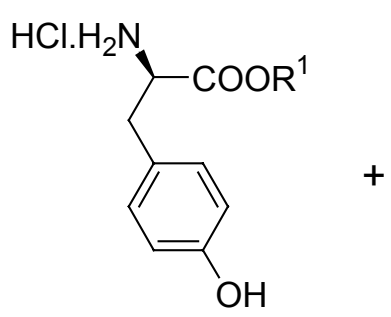

1
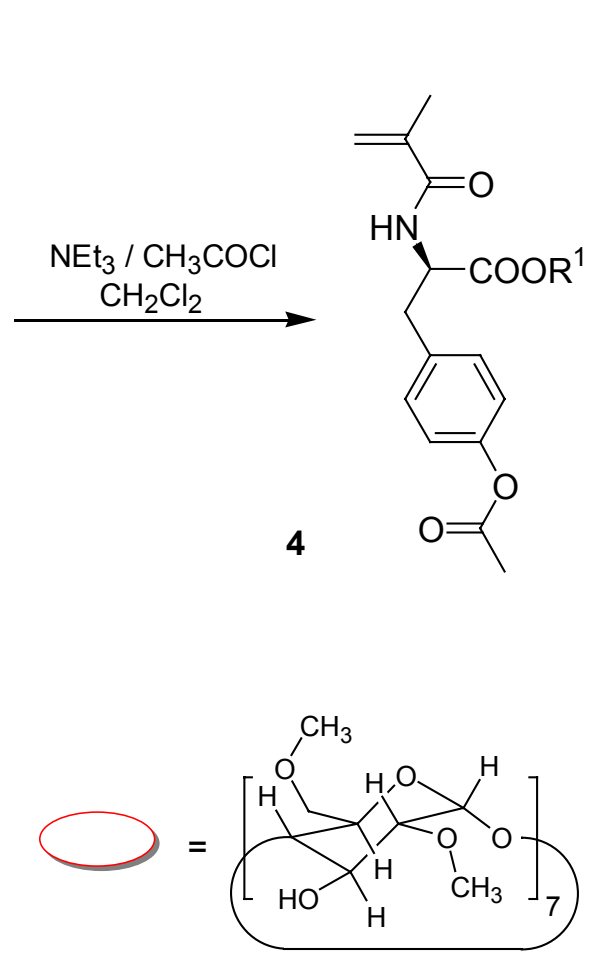

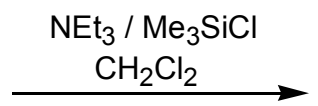

3
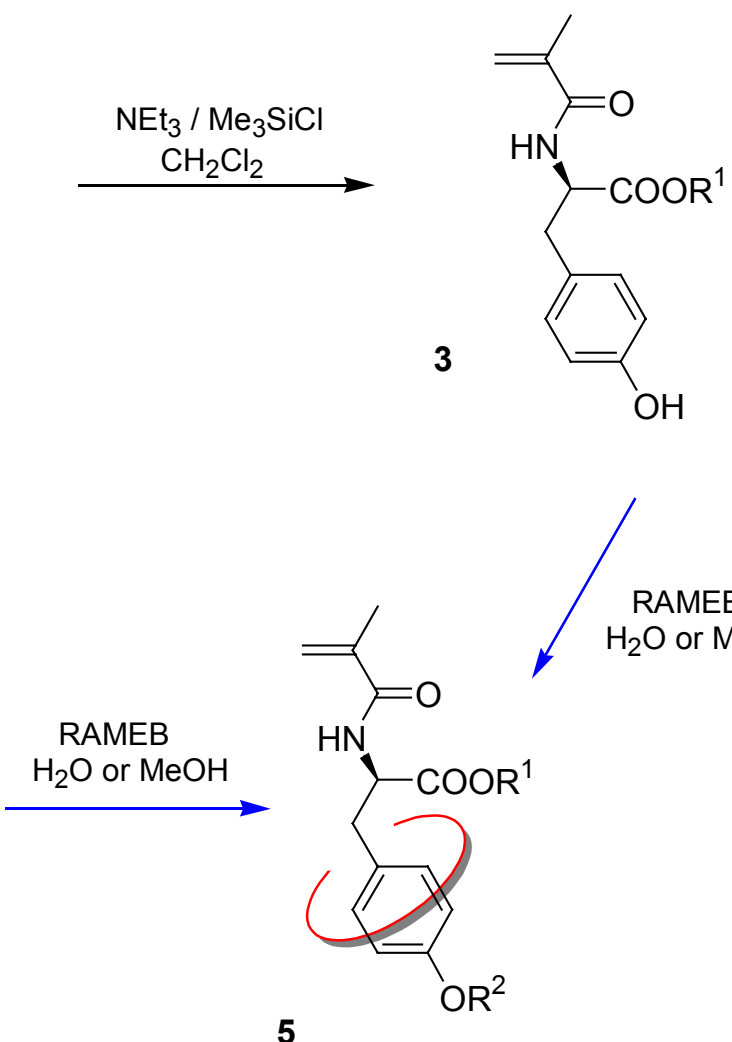

\begin{tabular}{l|ll} 
& $\mathrm{R}^{1}$ & $\mathrm{R}^{2}$ \\
\cline { 2 - 3 } $\mathbf{1 a} \mathbf{1}, \mathbf{3 a}, \mathbf{5 a}$ & $\mathrm{CH}_{3}$ & $\mathrm{H}$ \\
$\mathbf{1 b} \mathbf{3 b} \mathbf{5 b}$ & $\mathrm{C}_{2} \mathrm{H}_{5}$ & $\mathrm{H}$ \\
$\mathbf{4 a}, \mathbf{5 c}$ & $\mathrm{CH}_{3}$ & $\mathrm{COCH}_{3}$ \\
$\mathbf{4 b} \mathbf{5 d}$ & $\mathrm{C}_{2} \mathrm{H}_{5}$ & $\mathrm{COCH}_{3}$
\end{tabular}

Scheme 1. Synthesis of $N$-methacryloyl-L-tyrosine derivatives $\mathbf{3 a}, \mathbf{3} \mathbf{b}, \mathbf{4 a}, \mathbf{4} \mathbf{b}$, and their water soluble complexes $\mathbf{5 a - d}$ 


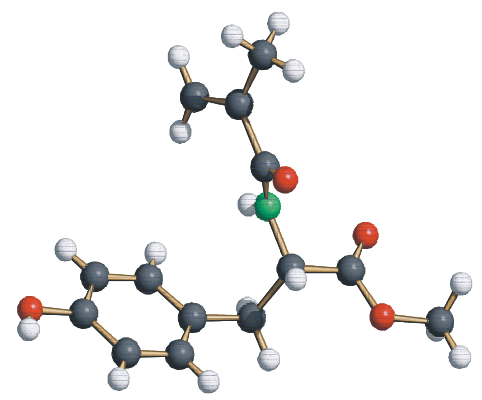

$3 a$

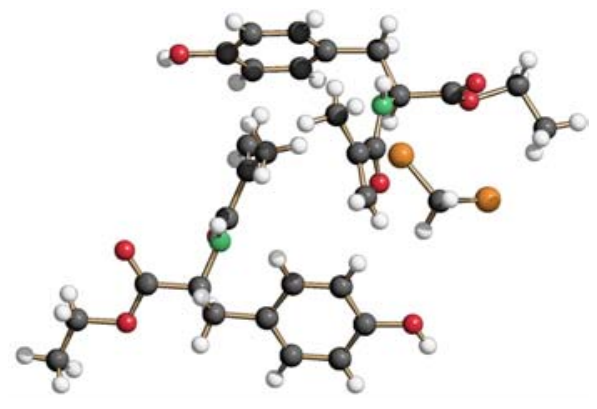

3b

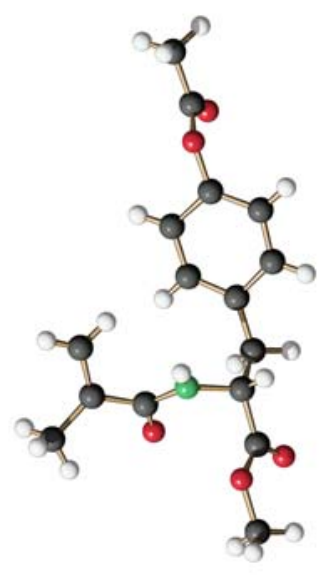

$4 a$

Fig. 1. Structures of $\mathbf{3 a}, \mathbf{3 b}$ and $\mathbf{4 a}$ obtained by X-ray analysis

The cyclodextrin host-guest complexes 5a-d were simply prepared in water (or methanol) by stirring an equimolar amount of RAMEB and the corresponding monomers (Scheme 1). The water-soluble complexes of the $N$-methacryloyl- $L$ tyrosine derivatives were isolated quantitatively after solvent evaporation. The formation of complexes was verified by thin layer chromatography, followed by UV detection and $\mathrm{I}_{2}$ development, 2D ROESY-NMR, and ${ }^{1} \mathrm{H}$ NMR spectroscopy. The $R_{\mathrm{f}}$ values of the uncomplexed monomers $3 \mathbf{a}, \mathbf{3 b}, \mathbf{4 a}$ and $\mathbf{4 b}$ are significantly higher compared to those of the complexes 5a-d (Tab. 1).

Tab. 1. $R_{\mathrm{f}}$ Values of uncomplexed monomers $\mathbf{3} \mathbf{a}, \mathbf{3 b}, \mathbf{4 a}, \mathbf{4} \mathbf{b}$ and RAMEB complexes 5a-d in methanol

\begin{tabular}{c|ccccccccc} 
& RAMEB & $\mathbf{3 a}$ & $\mathbf{5 a}$ & $\mathbf{3 b}$ & $\mathbf{5 b}$ & $\mathbf{4 a}$ & $\mathbf{5 c}$ & $\mathbf{4 b}$ & $\mathbf{5 d}$ \\
\hline$R_{\mathrm{f}}$ & 0.65 & 0.85 & 0.50 & 0.78 & 0.42 & 0.92 & 0.53 & 0.86 & 0.54
\end{tabular}

${ }^{1} \mathrm{H}$ NMR spectra of monomers $\mathbf{3} \mathbf{a}$ and $\mathbf{3} \mathbf{b}$ and the corresponding cyclodextrin complexes $\mathbf{5 a}$ and $\mathbf{5 b}$ were recorded in deuterium oxide $\left(\mathrm{D}_{2} \mathrm{O}\right)$. Because of the poor solubility of the uncomplexed monomers $4 a$ and $4 b$ in $\mathrm{D}_{2} \mathrm{O}$, we recorded the ${ }^{1} \mathrm{H}$ NMR measurement in a mixture of $\mathrm{D}_{2} \mathrm{O} /$ methanol- $d_{4}(5: 2 \mathrm{v} / \mathrm{v})$. Some significant differences in the shifts of the complexed and uncomplexed monomers are summarized in Tab. 2.

Fig. 2 shows the ${ }^{1} \mathrm{H}$ NMR spectra of monomer $3 \mathbf{a}$ and the heptakis(2,6-di-O-methyl)$\beta$-cyclodextrin DIMEB complex $5 \mathrm{a}$ in $\mathrm{D}_{2} \mathrm{O}$ as an example. While the aliphatic protons $\left(-\mathrm{CH},-\mathrm{CH}_{2}\right)$ and the ester group are not affected by the CD-torus, the signals of aromatic protons in ortho position to an $\mathrm{OH}$ group are significantly shifted to higher field, from 6.851 to $6.683 \mathrm{ppm}$. Obviously, in the complex 5a the signals of the methacryl groups $\mathrm{H}_{\mathrm{b}}$ (from 5.574 to $5.668 \mathrm{ppm}$ ), $\mathrm{H}_{\mathrm{a}}$ (from 5.429 to $5.474 \mathrm{ppm}$ ) and $\mathrm{CH}_{3}$ (from 1.845 to $1.911 \mathrm{ppm}$ ) were shifted to a lower magnetic field. The signal of the aromatic protons in meta position to an $\mathrm{OH}$ group shows a slightly positive difference in the magnetic shift. We assume that the vinyl group as well as the phenyl rest is situated 
in the torus of cyclodextrin. The exact position of the guest in the cyclodextrin cavity cannot be derived from the interpretation of the shifts in ${ }^{1} \mathrm{H}$ NMR signals.

Tab. 2. Shift in ${ }^{1} \mathrm{H}$ NMR spectra of monomers $\mathbf{3}$ and $\mathbf{4}$ and host-guest complexes $\mathbf{5}$.

1) $300 \mathrm{MHz}{ }^{1} \mathrm{H}$ NMR in $\mathrm{D}_{2} \mathrm{O} .{ }^{2)} 400 \mathrm{MHz}{ }^{1} \mathrm{H}$ NMR in $\mathrm{D}_{2} \mathrm{O} /$ methanol- $d_{4}(5: 2 \mathrm{v} / \mathrm{v})$<smiles>[R]OC(=O)[C@H](Cc1cc(C)c(OC(C)=O)cc1[2H])NC(=O)/C(C)=C/C</smiles>

\begin{tabular}{c|cccccc} 
& $\mathrm{H}_{\mathrm{m}}$ & $\mathrm{H}_{\mathrm{o}}$ & $\mathrm{H}_{\mathrm{b}}$ & $\mathrm{H}_{\mathrm{a}}$ & $\begin{array}{c}\mathrm{CH}_{3}- \\
\text { acetate }\end{array}$ & $\mathrm{CH}_{3}$-vinyl \\
\hline $\mathbf{3 a}^{1)}$ & 7.144 & 6.851 & 5.574 & 5.429 & - & 1.845 \\
$\mathbf{5 a}^{1)}$ & 7.117 & 6.683 & 5.668 & 5.474 & - & 1.911 \\
$\Delta$ & 0.027 & 0.168 & -0.094 & -0.045 & - & -0.066 \\
$\mathbf{3 b}^{1)}$ & 7.151 & 6.851 & 5.577 & 5.429 & - & 1.849 \\
$\mathbf{5 b}^{1)}$ & 7.112 & 6.697 & 5.671 & 5.472 & - & 1.912 \\
$\Delta$ & 0.039 & 0.154 & -0.094 & -0.043 & - & -0.063 \\
$\mathbf{4 a}^{2)}$ & 7.027 & 6.797 & 5.322 & 5.164 & 2.048 & 1.585 \\
$\mathbf{5 c}^{2)}$ & 7.063 & 6.785 & 5.366 & 5.208 & 2.041 & 1.621 \\
$\Delta$ & -0.036 & 0.012 & -0.044 & -0.044 & 0.007 & -0.036 \\
$\mathbf{4 b}^{2)}$ & 7.034 & 6.805 & 5.342 & 5.176 & 2.053 & 1.599 \\
$\mathbf{5 d}^{2)}$ & 7.078 & 6.797 & 5.378 & 5.222 & 2.040 & 1.636 \\
$\Delta$ & -0.044 & 0.008 & -0.036 & -0.046 & 0.013 & -0.037
\end{tabular}
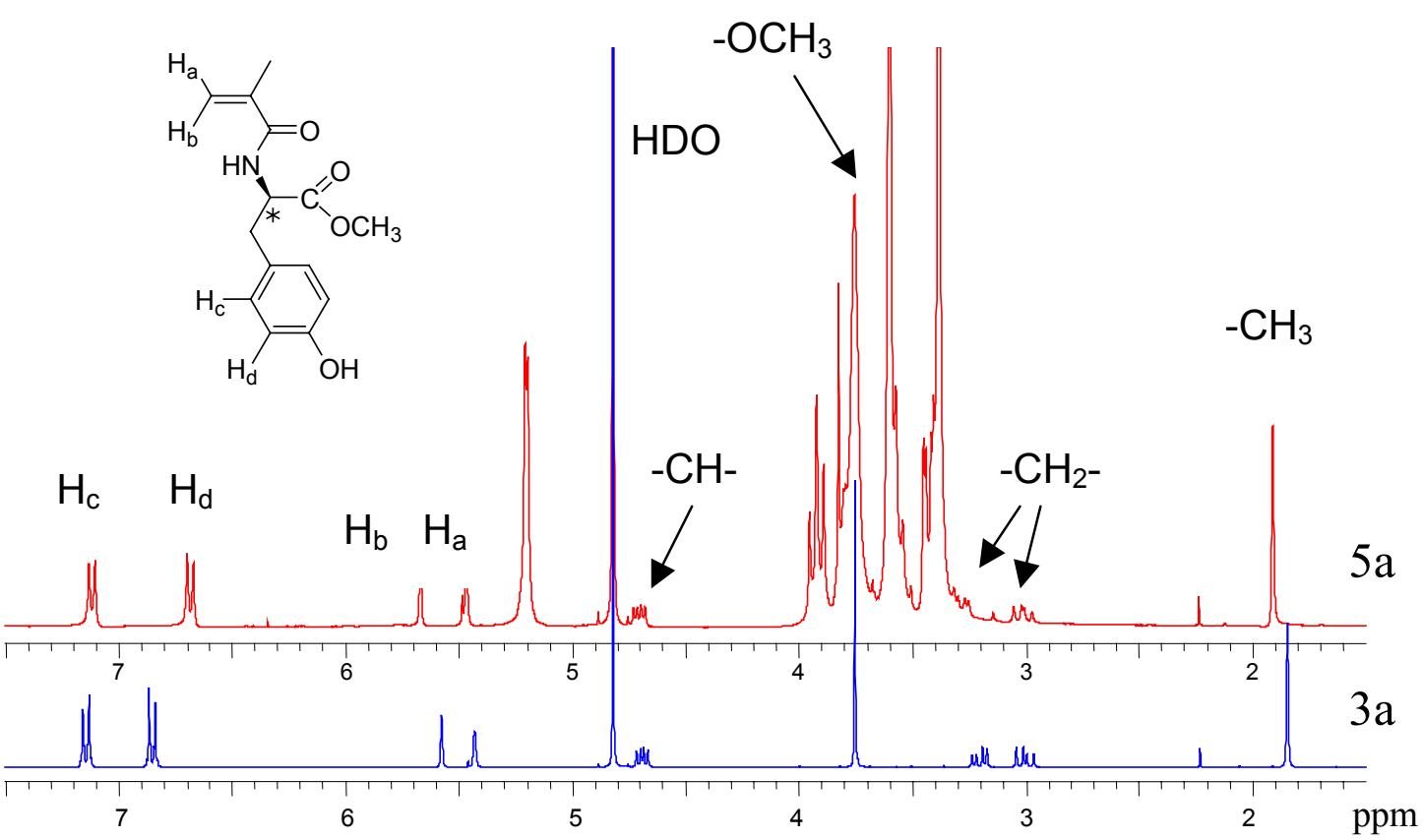

Fig. 2. $300 \mathrm{MHz}{ }^{1} \mathrm{H}$ NMR spectra of uncomplexed monomer 3a and complexed monomer $\mathbf{5 a}$ in $\mathrm{D}_{2} \mathrm{O}$ at room temperature 

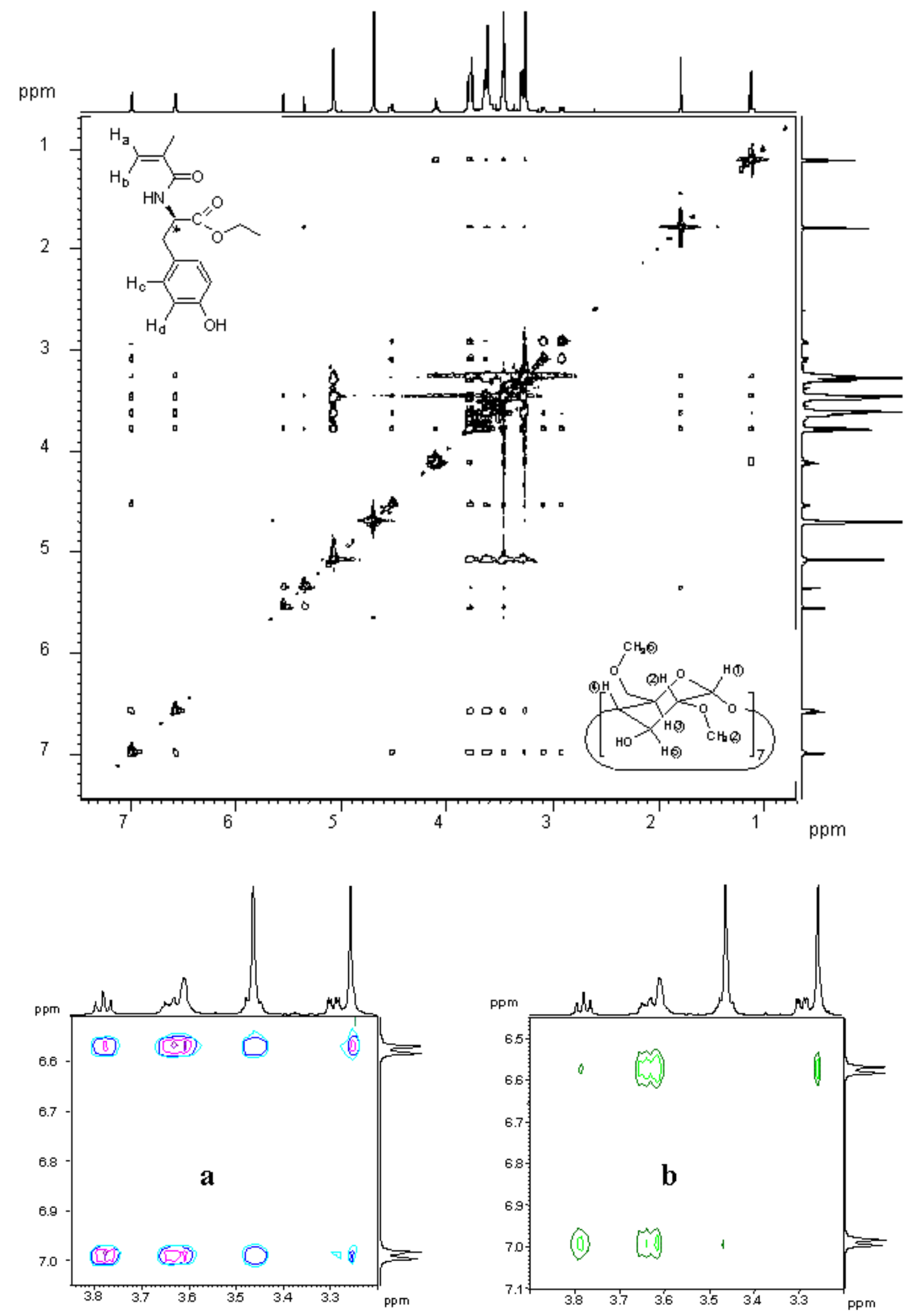

Fig. 3. $600 \mathrm{MHz}{ }^{1} \mathrm{H},{ }^{1} \mathrm{H}$ ROESY-NMR spectrum of $\mathbf{5 b}$ in $\mathrm{D}_{2} \mathrm{O}$. Region of aromatic protons: (a) $600 \mathrm{MHz}{ }^{1} \mathrm{H},{ }^{1} \mathrm{H}$-ROESY-NMR, (b) $600 \mathrm{MHz}{ }^{1} \mathrm{H},{ }^{1} \mathrm{H}$ NOESY-NMR 
Fig. 3 and $3 a$ show the 2D-ROESY-NMR spectrum of complex $5 \mathbf{b}$ in $\mathrm{D}_{2} \mathrm{O}$. The significant cross peaks confirm a host-guest interaction of aromatic protons $\mathrm{H}_{\mathrm{c}}$ and $\mathrm{H}_{\mathrm{d}}$ and the $\mathrm{H}(3), \mathrm{H}(5)$ and methoxy groups $\mathrm{OCH}_{3}(2)$ of cyclodextrin. Additionally, the aromatic proton in ortho position to $\mathrm{OH}$ groups of $\mathbf{3 b}$ interact with the methoxy groups $\mathrm{OCH}_{3}(6)$ of cyclodextrin. The cross peaks of the protons at the vinyl double bond $\left(\mathrm{H}_{\mathrm{a}}\right.$, $\mathrm{H}_{\mathrm{b}}$ and the methyl groups) and cyclodextrin are also smaller in comparison to the aromatic protons. The 2D-NOESY-NMR spectrum of $5 \mathbf{b}$ (Fig. $3 \mathrm{~b}$ ) confirmed the strong interaction of the aromatic protons $\mathrm{H}_{\mathrm{d}}$ with $\mathrm{OCH}_{3}(6)$ of the host compound. No cross peaks in the 2D-NOESY spectrum were found for the vinyl groups. According to the 2D-NMR spectra, it can be concluded that the aromatic moiety is located at the smaller part of the cyclodextrin torus. Additional X-ray analyses would be necessary in order to be able to determine the structure of the host-guest complex. So far we have not yet been able to grow a single crystal of this host-guest complex.

\section{Radical homopolymerization}

The host-guest complexes 5a-d were polymerized in aqueous medium at various temperatures. At first, the radical polymerizations of the water soluble complexes 5a-d were intended to be carried out in water at room temperature $\left(20-21^{\circ} \mathrm{C}\right)$ in the presence of a redox initiator. Surprisingly, the expected formation of polymeric precipitate was not observed during decomposition of the initiator. The reaction mixture remained clear for up to five days. This observation suggests that, at ambient temperature, a stable host-guest complex of the monomers exists, wherein the vinyl groups are preferentially located in the center of the cyclodextrin cavity. The voluminous L-tyrosine guest molecules form very stable complexes with cyclodextrin, which leads to a decrease in mobility. Due to these reduced dynamics, the free radical reaction with the double bond is hindered.

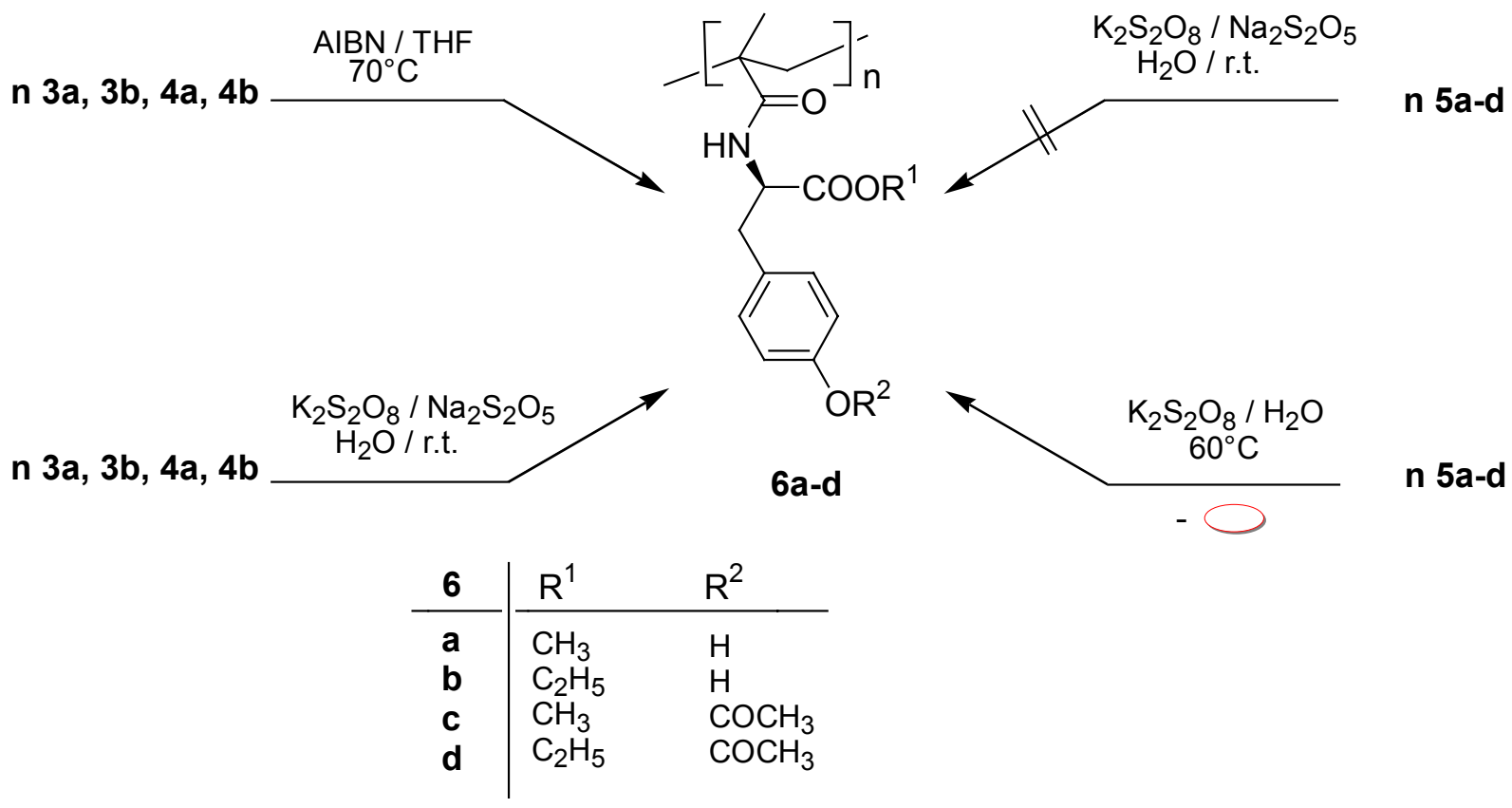

Scheme 2. Radical polymerization of free monomers $\mathbf{3 a}, \mathbf{3 b}, \mathbf{4} \mathbf{a}$ and $\mathbf{4 b}$ as well as RAMEB-complexes 5a-d 
At higher temperature (above $40^{\circ} \mathrm{C}$ ) the mobility of the guest monomer is increased, which leads to rapid polymerization of the water soluble complexes 5 a-d in aqueous medium (Scheme 2). During the polymerization, precipitation of the corresponding water-insoluble polymers $\mathbf{6 a - d}$ was observed from the first stages of the reaction. The polymers could be separated simply by filtration. It was proved by ${ }^{1} \mathrm{H}$ NMR spectroscopy and size exclusion chromatography (SEC) that all polymers 6a-d were not free of cyclodextrin, and about $5 \%(\mathrm{w} / \mathrm{w})$ of RAMEB still remained in the polymers. It was shown that in these cases the polymerization ends up with higher molecular weights than the polymerization of the uncomplexed monomers in water and also in organic solvent (Tab. 3). The SEC analysis of polymers 6a-d obtained from the complexed monomers 5a-d at $60^{\circ} \mathrm{C}$ showed a multimodal SEC curve. The polydispersities $\left(M_{\mathrm{w}} / M_{\mathrm{n}}\right)$ of polymers $6 \mathbf{a}-\mathbf{d}$ obtained from free radical polymerization of $5 \mathrm{a}-\mathbf{d}$ were higher than those of polymers obtained from uncomplexed monomers $\mathbf{3 a}, \mathbf{3 b}, \mathbf{4 a}$ and $\mathbf{4 b}$ in organic solvents.

Tab. 3. Yields and SEC results of polymers $\mathbf{6 a - d}$

\begin{tabular}{ccccc}
\hline Entry & $M_{\mathrm{n}}$ & $M_{\mathrm{w}}$ & $M_{\mathrm{w}} / M_{\mathrm{n}}$ & Yield in \% \\
\hline
\end{tabular}

Polymerization of cyclodextrin-complexed monomers in water at $60^{\circ} \mathrm{C}$ :

$\begin{array}{llrll}\text { 6a } & 22300 & 106300 & 4.8 & 70 \\ \mathbf{6 b} & 23800 & 95700 & 4.0 & 90 \\ \mathbf{6 c} & 17200 & 54100 & 3.1 & 57 \\ \mathbf{6 d} & 15500 & 64100 & 4.1 & 70\end{array}$

Polymerization of uncomplexed monomers in water at room temperature:

$\begin{array}{lrrrr}\text { 6a } & 14900 & 39500 & 2.6 & 63 \\ \mathbf{6 b} & 19200 & 59500 & 3.1 & 77 \\ \mathbf{6 c} & 8200 & 17100 & 2.1 & 50 \\ \mathbf{6 d} & 12000 & 25500 & 2.1 & 80\end{array}$

Polymerization of uncompelxed monomers in DMF at $70^{\circ} \mathrm{C}$ :

$\begin{array}{lllll}\mathbf{6 a} & 7200 & 16000 & 2.2 & 15 \\ \mathbf{6 b} & 7500 & 19200 & 2.6 & 70 \\ \mathbf{6 c} & 5700 & 17900 & 3.1 & 30 \\ \mathbf{6 d} & 6900 & 16200 & 2.3 & 20\end{array}$

Polymerization of uncomplexed monomers in tetrahydrofuran at $70^{\circ} \mathrm{C}$ :

$\begin{array}{llrll}\text { 6a } & 6200 & 13100 & 2.1 & 52 \\ \text { 6b } & 6300 & 17700 & 2.8 & 78 \\ \text { 6c } & 4000 & 9800 & 2.4 & 60 \\ \text { 6d } & 5400 & 9800 & 1.8 & 77\end{array}$

SEC results were obtained using $N, N$-dimethylformamide (DMF) as eluent with polystyrene as standard.

For comparison, the uncomplexed monomers 3 and 4 were polymerized in water at room temperature and also in an organic solvent, e.g., tetrahydrofuran (THF) or DMF (Tab. 3). Thus, the polymerization of a suspension of the uncomplexed monomers $3 \mathbf{a}, 3 \mathbf{b}, \mathbf{4 a}$ and $\mathbf{4 b}$ in aqueous medium at room temperature $\left(20-21^{\circ} \mathrm{C}\right)$ leads to 
polymers 6a-d, which showed monomodal SEC curves. However, the molecular weights of the resulting polymers were lower in comparison to those obtained for polymers produced from the complexed monomers $5 \mathrm{a}-\mathbf{d}$ at $60^{\circ} \mathrm{C}$. As a model study, the uncomplexed monomers $\mathbf{3}$ and $\mathbf{4}$ were also polymerized in homogeneous organic solution (THF, DMF) using 2,2'-azoisobutyronitrile (AIBN) as initiator. The polymers 6a-d were isolated in $50-80 \%$ yield. The average $M_{\mathrm{w}}$ of polymers $\mathbf{6 a - d}$ obtained from the aqueous phase were about four times higher than those of the corresponding polymers obtained from organic solution.

\section{Conclusions}

From the present investigation it can be concluded that forming a complex with $\beta$-cyclodextrin derivatives increases the water solubility of the chiral phenolic compounds bearing a methacrylic moiety. Thus we can assume that due to their voluminous structure the monomers form much more stable complexes with cyclodextrin at room temperature in comparison to smaller monomers, e.g., styrene. The cyclodextrin host seems to act as a thermosensitive monomer-guest stabilizer at room temperature. The complexed methacryl-tyrosin monomer can be polymerized radically only at higher temperature. The polymerization of uncomplexed monomers in heterogeneous aqueous medium or in organic solution only leads to polymers with lower molecular weights. This type of optically active polymers may give interesting materials, e.g., for the development of chiral chromatographic columns [20-22].

\section{Experimental part}

\section{Materials and measurements}

All chemicals were obtained from Fluka and used without further purification. All solvents of p.a. quality were stored over molecular sieves of 3 or $4 \AA$. Technical solvents were distilled before use. Technical cyclodextrin (RAMEB, CAVASOL W7 M PHARMA, degree of methylation 1.8) was purchased from Wacker, and Heptakis (2,6-di-O-methyl)-ß-cyclodextrin (DIMEB) was purchased from Cyclolabs R\&Lab. LTD., Budapest, Hungary.

Silica gel with $30-60 \mu \mathrm{m}$ particle size (Baker) was used for flash chromatography. Melting points were measured on a Büchi melting point apparatus. All melting points are uncorrected. ${ }^{1} \mathrm{H}$ NMR spectra were recorded with Bruker AC200, AM400, and Varian VXR-300, and 2D spectra with a Bruker DRX-600 at room temperature. IR spectra were run on a Nicolet 5SXB or a 5DXC FTIR spectrophotometer. The rotation angles $(\alpha)$ were measured with a Perkin-Elmer 241 Polarimeter ( $\mathrm{Hg} 578$ and 546 $\mathrm{nm}$ ). Elemental analysis was carried out in the Microanalytical Laboratory of the Institute of Organic Chemistry, University of Mainz. SEC analysis were performed with a setup of the PSS company, using $N, N$-dimethylformamide (DMF) containing $0.1 \% \mathrm{LiBr}$ as eluent at $75^{\circ} \mathrm{C} .100 \mu \mathrm{l}$ were injected on the column arrangement of PSS: HEMA $10 \mu, 40,100$, and $3000 \AA$ porosity. A TSP UV-Vis detector and a Shodex differential refractometer RI 71 were used as detectors. The data were evaluated with PSS-WinGPC 6.1 software. X-ray structure analyses were conducted on a SMART CCD Bruker AXS (Mo-K $\left.{ }_{\alpha}\right)$ or a Turbo Cad4 Enraf Nonius Diffractometer $\left(\mathrm{Cu}-\mathrm{K}_{\alpha}\right)$. 


\section{Synthesis of N-methacryloyl-L-tyrosine derivatives}

The general procedure for the synthesis of the methyl and ethyl esters of $L$-tyrosine is given in all cases for the methyl ester as an example: To a suspension of $2.78 \mathrm{~g}$ $(12.0 \mathrm{mmol}) \mathrm{L}$-tyrosine methyl ester hydrochloride (1a) in $60 \mathrm{ml}$ of absolute methylene chloride, $4.05 \mathrm{~g}(40.0 \mathrm{mmol})$ of triethylamine and $2.87 \mathrm{~g}(26.4 \mathrm{mmol})$ of trimethylchlorosilane were added at $0{ }^{\circ} \mathrm{C}$. After $2 \mathrm{~h}, 1.27 \mathrm{~g}(12.0 \mathrm{mmol})$ of methacryloyl chloride (2) in $10 \mathrm{ml}$ absolute methylene chloride was added and the mixture was stirred at room temperature for $20 \mathrm{~h}$. The mixture was dissolved in $150 \mathrm{ml}$ of methylene chloride and extracted with $100 \mathrm{ml}$ of water. The resulting organic phase was washed with $100 \mathrm{ml}$ of $2 \mathrm{M}$ hydrochloric acid and $200 \mathrm{ml}$ of water and dried over magnesium sulfate. The methylene chloride solution was evaporated. The crude product was purified by chromatography using $\mathrm{CHCl}_{3} /$ acetone 10:1 .

\section{$N$-Methacryloyl-L-tyrosine methyl ester (3a):}

Yield: $89 \%$ of 3a. m.p.: $74-75^{\circ} \mathrm{C}$. $[\alpha]=+73.9\left(c=1 \mathrm{~g} / 100 \mathrm{ml}\right.$ in $\left.\mathrm{CHCl}_{3}\right)$.

${ }^{1} \mathrm{H}$ NMR $\left(300 \mathrm{MHz}, \mathrm{CDCl}_{3}\right): \delta=1.91\left(\mathrm{~s}, 3 \mathrm{H}, \mathrm{CH}_{2}=\mathrm{C}\left(\mathrm{CH}_{3}\right)\right), 2.99-3.15\left(2 \mathrm{dd},{ }^{3} \mathrm{~J}=5.6\right.$ and $5,8 \mathrm{~Hz},{ }^{2} \mathrm{~J}=14.0$ and $\left.13.9 \mathrm{~Hz}, 2 \mathrm{H}, \mathrm{CH}_{2}\right), 3.74\left(\mathrm{~s}, 3 \mathrm{H}, \mathrm{OCH}_{3}\right), 4.85-4.92(\mathrm{~m}, 1 \mathrm{H}$, $\mathrm{CH}) ; 5.34$ (dd, $\left.1 \mathrm{H}, \mathrm{CH}_{2}=\mathrm{C}\right), 5.68\left(\mathrm{t}, 1 \mathrm{H}, \mathrm{CH}_{2}=\mathrm{C}\right), 6.30\left(\mathrm{~d},{ }^{3} \mathrm{~J}_{\mathrm{CH}-\mathrm{NH}}=7.8 \mathrm{~Hz}, 1 \mathrm{H}, \mathrm{NH}\right)$, $6.62(\mathrm{~s}, 1 \mathrm{H}, \mathrm{OH}), 6.72\left(\mathrm{~d},{ }^{3} \mathrm{~J}=8.5 \mathrm{~Hz}, 2 \mathrm{H}, A_{r} H_{\text {ortho }}\right), 6.92\left(\mathrm{~d},{ }^{3} \mathrm{~J}=8.5 \mathrm{~Hz}, 2 \mathrm{H}, A_{r} H_{\text {meta }}\right.$ ).

${ }^{1} \mathrm{H}$ NMR (300 MHz, $\left.\mathrm{D}_{2} \mathrm{O}\right): \delta=1.85\left(\mathrm{t}, 3 \mathrm{H}, \mathrm{CH}_{2}=\mathrm{C}\left(\mathrm{CH}_{3}\right)\right), 2.96-3.04\left(\mathrm{dd},{ }^{2} \mathrm{~J}=13.9 \mathrm{~Hz}\right.$, $\left.{ }^{3} \mathrm{~J}=9.3 \mathrm{~Hz}, 1 \mathrm{H}, \mathrm{CH}_{2}\right), 3.17-3.24\left(\mathrm{dd},{ }^{2} \mathrm{~J}=13.9 \mathrm{~Hz},{ }^{3} \mathrm{~J}=5.7\right.$ and $5.8 \mathrm{~Hz}, 1 \mathrm{H}, \mathrm{CH}_{2}$ ), $3.75\left(\mathrm{~s}, 3 \mathrm{H}, \mathrm{OCH}_{3}\right), 4.67-4.72\left(\mathrm{dd},{ }^{3} \mathrm{~J}=5.7\right.$ and $\left.5.8 \mathrm{~Hz},{ }^{3} \mathrm{~J}=9.3 \mathrm{~Hz}, 1 \mathrm{H}, \mathrm{CH}\right), 5.48$, $5.67\left(\mathrm{bs}, 2 \mathrm{H}, \mathrm{CH}_{2}=\mathrm{C}\right), 6.85\left(\mathrm{~d},{ }^{3} \mathrm{~J}=8.6 \mathrm{~Hz}, 2 \mathrm{H}, \mathrm{ArH}_{\text {ortho }}\right), 7.14\left(\mathrm{~d},{ }^{3} \mathrm{~J}=8.6 \mathrm{~Hz}, 2 \mathrm{H}\right.$, $\mathrm{ArH}_{\text {meta }}$.

IR (diamond): $v / \mathrm{cm}^{-1}=3395(\mathrm{OH}), 3351(\mathrm{NH}), 3230$ (aryl) 2955, 2928, 2839 (alkyl), $1723(\mathrm{C}=\mathrm{O}), 1635$ (amide I), 1514 (amide II), $1446\left(-\mathrm{CH}_{2},-\mathrm{CH}_{3}\right), 1370$ (-C-O-), 1196 (-C-O-C-), 826 (1,4-disubstituted aromatic ring), further intensive signals: 1614, 1596, 1159, 1184, 1020, 964, 852.

$\begin{array}{llllllll}\mathrm{C}_{14} \mathrm{H}_{17} \mathrm{NO}_{4} \text { (263.29) } & \text { Calc. } & \mathrm{C} & 63.87 & \mathrm{H} & 6.51 & \mathrm{~N} & 5.32 \\ & \text { Found } & \mathrm{C} & 63.79 & \mathrm{H} & 6.56 & \mathrm{~N} & 5.26\end{array}$

Crystal data $\left(\mathrm{CH}_{2} \mathrm{Cl}_{2}\right.$ /petroleum ether): $\mathrm{C}_{14} \mathrm{H}_{17} \mathrm{NO}_{4}+0.5 \mathrm{CH}_{2} \mathrm{Cl}_{2}$; temperature of measurement: $-90^{\circ} \mathrm{C}$; space group $=\mathrm{P} 21212$ (orthorhombic); unit cell dimension: $a=$ 13.9703(10) $\AA, b=14.6943(11) \AA, c=15.7323(12) \AA ; V=3229.6(7) \AA^{3} ; z=8, F(000)$ $=1288 ; d=1.258 \mathrm{~g} \cdot \mathrm{cm}^{-3}$.

$N$-Methacryloyl-L-tyrosine ethyl ester (3b):

Yield: $73 \%$ of 3b. mp.: $71-72^{\circ} \mathrm{C}$. $[\alpha]=+66.8\left(c=1 \mathrm{~g} / 100 \mathrm{ml}\right.$ in $\left.\mathrm{CHCl}_{3}\right)$.

${ }^{1} \mathrm{H}$ NMR $\left(300 \mathrm{MHz}, \mathrm{CDCl}_{3}\right): \delta=1.26\left(\mathrm{t},{ }^{3} \mathrm{~J}=7.1 \mathrm{~Hz}, 3 \mathrm{H}, \mathrm{OCH}_{2} \mathrm{CH}_{3}\right), 1.91(\mathrm{~s}, 3 \mathrm{H}$, $\left.\mathrm{CH}_{2}=\mathrm{C}\left(\mathrm{CH}_{3}\right)\right), 2.99-3.15\left(2 \mathrm{dd},{ }^{3} \mathrm{~J}=5.6\right.$ and $\left.5,8 \mathrm{~Hz},{ }^{2} \mathrm{~J}=14.0 \mathrm{~Hz}, 2 \mathrm{H}, \mathrm{CH}_{2}\right), 4.19$ (q, $\left.{ }^{3} \mathrm{~J}=7.1 \mathrm{~Hz}, 2 \mathrm{H}, \mathrm{OCH}_{2} \mathrm{CH}_{3}\right), 4.83-4.90(\mathrm{~m}, 1 \mathrm{H}, \mathrm{CH}) ; 5.34\left(\mathrm{dd}, 1 \mathrm{H}, \mathrm{CH}_{2}=\mathrm{C}\right), 5.68(\mathrm{t}$, $\left.1 \mathrm{H}, \mathrm{CH}_{2}=\mathrm{C}\right), 6.31\left(\mathrm{~d},{ }^{3} \mathrm{~J}_{\mathrm{CH}-\mathrm{NH}}=7.8 \mathrm{~Hz}, 1 \mathrm{H}, \mathrm{NH}\right), 6.51(\mathrm{~s}, 1 \mathrm{H}, \mathrm{OH}), 6.71\left(\mathrm{~d},{ }^{3} \mathrm{~J}=8.5\right.$ $\mathrm{Hz}, 2 \mathrm{H}$, ArH $\left._{\text {ortho }}\right), 6.93\left(\mathrm{~d},{ }^{3} \mathrm{~J}=8.5 \mathrm{~Hz}, 2 \mathrm{H}\right.$, ArH $\left._{\text {meta }}\right)$.

${ }^{1} \mathrm{H}$ NMR $\left(300 \mathrm{MHz}, \mathrm{D}_{2} \mathrm{O}\right): \delta=1.22\left(\mathrm{t}, J=7.1 \mathrm{~Hz}, 3 \mathrm{H}, \mathrm{OCH}_{2} \mathrm{CH}_{3}\right), 1.85(\mathrm{~s}, 3 \mathrm{H}$, $\left.\mathrm{CH}_{2}=\mathrm{C}\left(\mathrm{CH}_{3}\right)\right), 2.98-3.06\left(\mathrm{dd},{ }^{2} \mathrm{~J}=13.9 \mathrm{~Hz},{ }^{3} \mathrm{~J}=9.0 \mathrm{~Hz}, 1 \mathrm{H}, \mathrm{CH}_{2}\right.$ ), $3.15-3.22\left(\mathrm{dd},{ }^{2} \mathrm{~J}\right.$ 
$\left.=13.9 \mathrm{~Hz},{ }^{3} \mathrm{~J}=6.23 \mathrm{~Hz}, 1 \mathrm{H}, \mathrm{CH}_{2}\right), 4.15-4.22\left(2 \mathrm{q}, 2 \mathrm{H},{ }^{3} \mathrm{~J}=7.1\right.$ and $7.3 \mathrm{~Hz}$, $\left.\mathrm{OCH}_{2} \mathrm{CH}_{3}\right), 4.64,4.65\left(2 \mathrm{~d},{ }^{3} \mathrm{~J}=9.0\right.$ and $\left.8.8 \mathrm{~Hz}, 1 \mathrm{H}, \mathrm{CH}\right), 5.43,5.58$ (bs, $2 \mathrm{H}, \mathrm{CH}_{2}=\mathrm{C}$ ), $6.85\left(\mathrm{~d},{ }^{3} \mathrm{~J}=8.6 \mathrm{~Hz}, 2 \mathrm{H}, A r \mathrm{Ar}_{\text {ortho }}\right), 7.15\left(\mathrm{~d},{ }^{3} \mathrm{~J}=8.6 \mathrm{~Hz}, 2 \mathrm{H}, \mathrm{ArH}_{\text {meta }}\right)$.

IR (diamond): $v / \mathrm{cm}^{-1}=3443(\mathrm{OH}), 3273(\mathrm{NH}), 3073$ (aryl), 2949, 2924, 2903, 2858 (alkyl), $1724(\mathrm{C}=\mathrm{O}), 1653$ (amide I), $1623(-\mathrm{C}=\mathrm{C}-), 1515$ (amide II), $1447\left(-\mathrm{CH}_{2}\right.$, $\left.-\mathrm{CH}_{3}\right), 1267$ (-C-O-), 826 (1,4-disubstituted aromatic ring), further intensive signals: $1541,1372,1207,1170,1112,1021,912,874$.

$\begin{array}{llllllll}\mathrm{C}_{15} \mathrm{H}_{19} \mathrm{NO}_{4} \text { (277.32) } & \text { Calc. } & \mathrm{C} & 4.97 & \mathrm{H} & 6.91 & \mathrm{~N} & 5.05 \\ & \text { Found } & \mathrm{C} & 64.07 & \mathrm{H} & 6.79 & \mathrm{~N} & 4.76\end{array}$

Crystal data $\left(\mathrm{CH}_{2} \mathrm{Cl}_{2}\right.$ /petroleum ether): $\mathrm{C}_{15} \mathrm{H}_{19} \mathrm{NO}_{4}+0.5 \mathrm{CH}_{2} \mathrm{Cl}_{2}$; temperature of measurement: $22^{\circ} \mathrm{C}$; space group $=\mathrm{P} 2{ }_{1} 212$ (orthorhombic); unit cell dimension: $a=$ 14.3283(6) $\AA, b=15.1414(5) \AA, c=16.1115(10) \AA ; V=3495.4(3) \AA^{3} ; z=8, F(000)=$

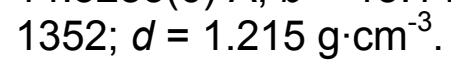

\section{Acetylation of N-methacryloyl-L-tyrosine derivatives}

$2.00 \mathrm{~g}(7.6 \mathrm{mmol})$ of $3 \mathrm{a}$ and $0.77 \mathrm{~g}(7.6 \mathrm{mmol})$ of triethylamine were dissolved in 80 $\mathrm{ml}$ of absolute methylene chloride. $0.6 \mathrm{~g}(7.6 \mathrm{mmol})$ of acetyl chloride was added dropwise to the solution at $0^{\circ} \mathrm{C}$. The clear solution was stirred at room temperature for 3 days. The solution was washed with $100 \mathrm{ml}$ of $2 \mathrm{M}$ hydrochloric acid. The organic phase was separated, washed with water and dried over magnesium sulfate. Methylene chloride was removed by evaporation. The slightly yellow product was crystallized in a refrigerator. The crude product was pure enough for further use. An analytical sample was purified by chromatography with $\mathrm{CHCl}_{3} /$ acetone $(10: 1 \mathrm{v} / \mathrm{v})$ for further evaluation.

\section{O-Acety- $N$-methacrylol-L-tyrosine methyl ester (4a):}

Yield: $50-70 \%$ of 4 a. m.p.: $96-97^{\circ} \mathrm{C}$.

${ }^{1} \mathrm{H}$ NMR $\left(200 \mathrm{MHz}, \mathrm{CDCl}_{3}\right): \delta=1.90\left(\mathrm{~s}, 3 \mathrm{H}, \mathrm{CH}_{2}=\mathrm{C}\left(\mathrm{CH}_{3}\right)\right), 2.26\left(\mathrm{~s}, 3 \mathrm{H}, \mathrm{C}(\mathrm{O}) \mathrm{CH}_{3}\right)$, $3.14\left(\mathrm{~d},{ }^{3} \mathrm{~J}_{\mathrm{CH}-\mathrm{CH} 2}=5.86 \mathrm{~Hz}, 1 \mathrm{H}, \mathrm{CH}_{2}\right), 3.16\left(\mathrm{~d},{ }^{3} \mathrm{~J}_{\mathrm{CH}-\mathrm{CH} 2}=5.85 \mathrm{~Hz}, 1 \mathrm{H}, \mathrm{CH}_{2}\right), 3.2(\mathrm{~s}$, $\left.3 \mathrm{H}, \mathrm{OCH}_{3}\right), 4.94-4.84\left(2 \mathrm{dd},{ }^{3} J_{\mathrm{CH}-\mathrm{NH}}=7.32 \mathrm{~Hz},{ }^{3} \mathrm{~J}_{\mathrm{CH}-\mathrm{CH} 2}=5.86 \mathrm{~Hz}, 1 \mathrm{H}, \mathrm{CH}\right), 5.33(\mathrm{~s}$, $\left.1 \mathrm{H}, \mathrm{CH}_{2}=\mathrm{C}\right), 5.64\left(\mathrm{~s}, 1 \mathrm{H}, \mathrm{CH}_{2}=\mathrm{C}\right), 6.22\left(\mathrm{~d},{ }^{2} \mathrm{~J}_{\mathrm{CH}-\mathrm{NH}}=7.32 \mathrm{~Hz}, 1 \mathrm{H}, \mathrm{NH}\right), 6.98\left(\mathrm{~d},{ }^{3} \mathrm{~J}=\right.$ $8.30 \mathrm{~Hz}, 2 \mathrm{H}$, ArH $\left._{\text {ortho }}\right), 7.08$ (d, ${ }^{3} \mathrm{~J}=8.78 \mathrm{~Hz}, 2 \mathrm{H}$, ArH $_{\text {meta }}$ ).

${ }^{1} \mathrm{H}$ NMR $\left(200 \mathrm{MHz}, \mathrm{D}_{2} \mathrm{O} /\right.$ methanol- $\left.d_{4}, 5: 2 \mathrm{v} / \mathrm{v}\right): \delta=1.59\left(\mathrm{~s}, 3 \mathrm{H}, \mathrm{CH}_{2}=\mathrm{C}\left(\mathrm{CH}_{3}\right)\right), 2.05(\mathrm{~s}$, $\left.3 \mathrm{H}, \mathrm{C}(\mathrm{O}) \mathrm{CH}_{3}\right), 2.75-2.99\left(\mathrm{~m}, 2 \mathrm{H}, \mathrm{CH}_{2}\right), 3.48\left(\mathrm{~s}, 3 \mathrm{H}, \mathrm{OCH}_{3}\right), 4.41-4.49(2 \mathrm{~d}, 1 \mathrm{H}$, $\mathrm{CH}), 5.16\left(\mathrm{~s}, 1 \mathrm{H}, \mathrm{CH}_{2}=\mathrm{C}\right), 5.32\left(\mathrm{~s}, 1 \mathrm{H}, \mathrm{CH}_{2}=\mathrm{C}\right), 6.78\left(\mathrm{~d},{ }^{3} \mathrm{~J}=8.30 \mathrm{~Hz}, 2 \mathrm{H}, \mathrm{ArH}_{\text {ortho }}\right)$, $7.03\left(\mathrm{~d},{ }^{3} \mathrm{~J}=8.30 \mathrm{~Hz}, 2 \mathrm{H}, \mathrm{ArH}_{\text {meta }}\right)$.

IR (diamond): $v / \mathrm{cm}^{-1}=3316(\mathrm{NH}), 3062,3042$ (aryl), 2954, 2923, 2903, 2852 (alkyl), 1749, $1728(\mathrm{C}=\mathrm{O}), 1650$ (amide I), 1608 (-C=C-), 1510 (amide II), $1440\left(-\mathrm{CH}_{2},-\mathrm{CH}_{3}\right)$, 1221 (-C-O-), 835 (1,4-disubstituted benzenes), further intensive signals: 1536, 1373, 1346, 1285, 1221, 1173, 1105, 1033, 1014, 937, 920, 875.

$\begin{array}{llllllll}\mathrm{C}_{16} \mathrm{H}_{19} \mathrm{NO}_{5} \text { (305.15) } & \text { Calc. } & \mathrm{C} & 62.92 & \mathrm{H} & 6.28 & \mathrm{~N} & 4.59 \\ & \text { Found } & \mathrm{C} & 62.90 & \mathrm{H} & 6.33 & \mathrm{~N} & 4.57\end{array}$

Crystal data (cyclodextrin solution in $\mathrm{D}_{2} \mathrm{O}$ ): $\mathrm{C}_{16} \mathrm{H}_{19} \mathrm{NO}_{5}$, temperature of measurement: $22^{\circ} \mathrm{C}$; space group $=\mathrm{C}_{2}$ (orthorhombic); unit cell dimension: $a=17.955(27) \AA, b=$ 
5.046(3) $\AA, c=18.106(22) \AA ; \beta=98.47(6)^{\circ}, V=1623(3) \AA^{3} ; z=4, F(000)=648 ; d=$ $1.250 \mathrm{~g} \cdot \mathrm{cm}^{-3}$.

O-Acetyl-N-methacryloyl-L-tyrosine ethyl ester (4b):

The synthesis of $\mathbf{4 b}$ was carried out analogously to the procedure described above. The crude product was purified by chromatography using $\mathrm{CHCl}_{3} /$ acetone $(20: 1 \mathrm{v} / \mathrm{v})$. The pure compound $\mathbf{4 b}$ was crystallized in a refrigerator as a colourless powder. Yield: $51 \%$ of $\mathbf{4 b}$. m.p.: $65-66^{\circ} \mathrm{C}$

${ }^{1} \mathrm{H}$ NMR $\left(400 \mathrm{MHz}, \mathrm{CDCl}_{3}\right): \delta=1.22\left(\mathrm{t},{ }^{3} \mathrm{~J}=7.19 \mathrm{~Hz}, 3 \mathrm{H}, \mathrm{OCH}_{2} \mathrm{CH}_{3}\right), 1.89(\mathrm{~s}, 3 \mathrm{H}$, $\left.\mathrm{CH}_{2}=\mathrm{C}\left(\mathrm{CH}_{3}\right)\right), 2.25\left(\mathrm{~s}, 3 \mathrm{H}, \mathrm{C}(\mathrm{O}) \mathrm{CH}_{3}\right), 3.14-3.12\left(2 \mathrm{~d}, 2 \mathrm{H}, \mathrm{CH}_{2}\right), 4.15$ (q, ${ }^{3} \mathrm{~J}=7.14$ $\left.\mathrm{Hz}, 2 \mathrm{H}, \mathrm{OCH}_{2} \mathrm{CH}_{3}\right), 4.83-4.87\left(2 \mathrm{dd},{ }^{3} \mathrm{~J}_{\mathrm{CH}-\mathrm{NH}}=7.63 \mathrm{~Hz},{ }^{3} \mathrm{~J}_{\mathrm{CH}-\mathrm{CH} 2}=5.87 \mathrm{~Hz}, 1 \mathrm{H}, \mathrm{CH}\right)$, $5.31\left(\mathrm{~s}, 1 \mathrm{H}, \mathrm{CH}_{2}=\mathrm{C}\right) 5.64\left(\mathrm{~s}, 1 \mathrm{H}, \mathrm{CH}_{2}=\mathrm{C}\right), 6.27\left(\mathrm{~d},{ }^{3} J_{\mathrm{NH}-\mathrm{CH}}=7.33 \mathrm{~Hz}, 1 \mathrm{H}, \mathrm{NH}\right), 6.97$ (d, $\left.{ }^{3} \mathrm{~J}=8.51 \mathrm{~Hz}, 2 \mathrm{H}, \mathrm{ArH}_{\text {ortho }}\right) ; 7.08\left(\mathrm{~d},{ }^{3} \mathrm{~J}=8.51 \mathrm{~Hz}, 2 \mathrm{H}, \mathrm{ArH}_{\text {meta }}\right.$ ).

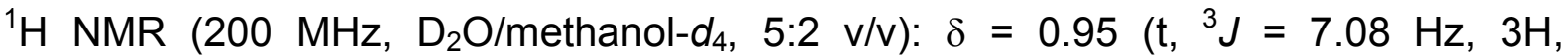
$\left.\mathrm{OCH}_{2} \mathrm{CH}_{3}\right), 1.60\left(\mathrm{~s}, 3 \mathrm{H}, \mathrm{CH}_{2}=\mathrm{C}\left(\mathrm{CH}_{3}\right)\right), 2.05\left(\mathrm{~s}, 3 \mathrm{H}, \mathrm{C}(\mathrm{O}) \mathrm{CH}_{3}\right), 2.92\left(\mathrm{~m}, 2 \mathrm{H}, \mathrm{CH}_{2}\right)$, $3.91\left(\mathrm{q},{ }^{3} \mathrm{~J}=7.00 \mathrm{~Hz}, 2 \mathrm{H}, \mathrm{OCH}_{2} \mathrm{CH}_{3}\right), 4.41(\mathrm{~m}, 1 \mathrm{H}, \mathrm{CH}), 5.18\left(\mathrm{~s}, 1 \mathrm{H}, \mathrm{CH}_{2}=\mathrm{C}\right) 5.34(\mathrm{~s}$, $1 \mathrm{H}, \mathrm{CH}_{2}=\mathrm{C}$ ), 6.80 (d, $\left.{ }^{3} \mathrm{~J}=8.30 \mathrm{~Hz}, 2 \mathrm{H}, \mathrm{ArH}_{\text {ortho }}\right) ; 7.03$ (d, ${ }^{3} \mathrm{~J}=8.30 \mathrm{~Hz}, 2 \mathrm{H}, \mathrm{ArH}_{\text {meta }}$ ).

IR (KBr): $v / \mathrm{cm}^{-1}=3319(\mathrm{NH}), 3064,3054,3042$ (aryl), 2980, 2955, 2936, 2871 (alkyl), 1750, 1740 (C=O), 1660 (amide I), 1618 (-C=C-), 1533 (amide II), 1447, 1372 $\left(-\mathrm{CH}_{2},-\mathrm{CH}_{3}\right), 850$ (1,4-disubstituted aromatic ring), further intensive signals: 1509, 1346, 1247, 1217, 1200, 1169, 1115, 1103, 1040, 1019, 941, 916.

$\begin{array}{llllllll}\mathrm{C}_{17} \mathrm{H}_{21} \mathrm{NO}_{5}(319.14) & \text { Calc. } & \mathrm{C} & 63.72 & \mathrm{H} & 6.61 & \mathrm{~N} & 4.37 \\ & \text { Found } & \mathrm{C} & 63.55 & \mathrm{H} & 6.30 & \mathrm{~N} & 4.33\end{array}$

\section{Synthesis of the guest-host complexes $\mathbf{5 a - d}$}

The general synthesis of host-guest complexes of L-tyrosine compounds is demonstrated in all cases for the RAMEB complex $5 \mathrm{c}$ as an example.

$3.8 \mathrm{mmol}$ of monomers $4 \mathrm{a}$ and $3.9 \mathrm{mmol}$ of RAMEB were stirred in $25 \mathrm{ml}$ methanol. The solvent was evaporated after $2 \mathrm{~h}$ and the water-soluble solid complex was dried in vacuum. Yield: $100 \%$ of the complex in all cases.

The NMR measurement in $\mathrm{D}_{2} \mathrm{O}$ was carried out using heptakis(2,6-di-O-methyl)- $\beta$ cyclodextrin (DIMEB).

\section{DIMEB complex of $3 \mathbf{a}(\mathbf{5 a})$ :}

${ }^{1} \mathrm{H}$ NMR (300 MHz, $\left.\mathrm{D}_{2} \mathrm{O}\right): \delta=1.91$ (s, 3H, $\left.\mathrm{CH}_{2}=\mathrm{CH}(\mathrm{CH} 3)\right), 2.97-3.05\left(\mathrm{~m}, 1 \mathrm{H}, \mathrm{CH}_{2}\right)$,

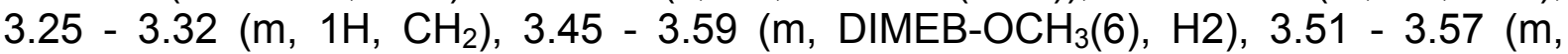
DIMEB-H4), 3.60 (DIMEB-OCH $\left.{ }_{3}(2)\right), 3.65$ - 3.86 (DIMEB-H6, H5), 3.83 (s, 3H, $\mathrm{OCH}_{3}$ ), 3.92 (t, DIMEB-H3), 4.17 - $4.73(2 \mathrm{~d}, 1 \mathrm{H}, \mathrm{CH}), 5.205$ (d, DIMEB-H1), 5.47 (s, $\left.\mathrm{H}, \mathrm{CH}_{2}=\mathrm{C}\right), 5.67\left(\mathrm{~s}, 1 \mathrm{H}, \mathrm{CH}_{2}=\mathrm{C}\right), 6.68\left(\mathrm{~d},{ }^{3} \mathrm{~J}=8.42 \mathrm{~Hz}, 2 \mathrm{H}, \mathrm{ArH}_{\text {ortho }}\right), 7.12$ (d, ${ }^{3} \mathrm{~J}=$ $\left.8.42 \mathrm{~Hz}, 2 \mathrm{H}, \mathrm{ArH}_{\mathrm{meta}}\right)$.

IR (diamond): $\mathrm{v}\left(\mathrm{cm}^{-1}\right)=3421$ (RAMEB-OH), 2930, 2836 (RAMEB-alkyl), 1739 $(\mathrm{C}=\mathrm{O}), 1664$ (amide I), 1616 (-C=C-), 1516 (amide II), 1450, $1364\left(\mathrm{RAMEB}-\mathrm{CH}_{2}\right.$, $-\mathrm{CH}_{3}$ ), 1197 (-C-O-C-), 1154, 1083, 1036, 965 (RAMEB -C-O-C-, -C-O-), 857 (1,4disubstituted aromatic ring). 


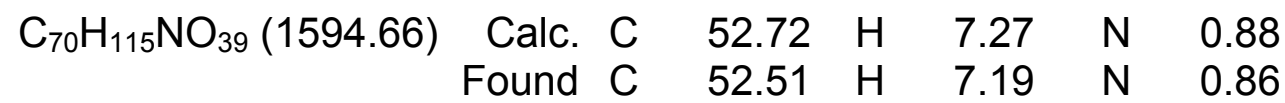

DIMEB complex of $\mathbf{3 b} \mathbf{b} \mathbf{5} \mathbf{b})$ :

${ }^{1} \mathrm{H}$ NMR $\left(300 \mathrm{MHz}, \mathrm{D}_{2} \mathrm{O}\right): \delta=1.23\left(\mathrm{t},{ }^{3} \mathrm{~J}=7.05 \mathrm{~Hz}, 3 \mathrm{H}, \mathrm{OCH}_{2} \mathrm{CH}_{3}\right), 1.91(\mathrm{~s}, 3 \mathrm{H}$, $\left.\mathrm{CH}_{2}=\mathrm{C}\left(\mathrm{CH}_{3}\right)\right), 3.00-3.23\left(\mathrm{~m}, 2 \mathrm{H}, \mathrm{CH}_{2}\right), 3.39(\mathrm{~s}$, DIMEB-OCH $(6)), 3.44-3.52(\mathrm{~m}$, DIMEB-H4, H2), 3.59 (s, DIMEB-OCH $\left.{ }_{3}(2)\right), 3.76-3.82$ (DIMEB-H5, H6), 3.91 (t, DIMEB-H3), $4.19-4.26\left(\mathrm{~m}, 2 \mathrm{H}, \mathrm{OCH}_{2} \mathrm{CH}_{3}\right), 4.64\left(\mathrm{~d},{ }^{3} \mathrm{~J}=8.97,1 \mathrm{H}, \mathrm{CH}\right), 4.66\left(\mathrm{~d},{ }^{3} \mathrm{~J}=\right.$ 8.97, $1 \mathrm{H}, \mathrm{CH}), 5.185,5.19$ (d, DIMEB-H1), 5.47, $5.67\left(\mathrm{~s}, 2 \mathrm{H}, \mathrm{CH}_{2}=\mathrm{C}\right), 6.70\left(\mathrm{~d},{ }^{3} \mathrm{~J}=\right.$ $8.24 \mathrm{~Hz}, 2 \mathrm{H}, \mathrm{ArH}_{\text {ortho }}$ ), 7.11 (d, ${ }^{3} \mathrm{~J}=8.28 \mathrm{~Hz}, 2 \mathrm{H}, \mathrm{ArH}_{\text {meta }}$ ).

IR (KBr): $v / \mathrm{cm}^{-1}=3412$ (RAMEB-OH), 2932 (RAMEB-Alkyl), 1735 (C=O), 1659 (amide I), 1517 (amide II), 1454, 1371 (RAMEB- $\mathrm{CH}_{2},-\mathrm{CH}_{3}$ ), 1220 (-C-O-C-), 1162, 1088, 1044 (RAMEB -C-O-C-, -C-O-), further intensive signal: 1627.

$\begin{array}{llllllll}\mathrm{C}_{71} \mathrm{H}_{117} \mathrm{NO}_{39}(1608.59) & \text { Calc. } & \mathrm{C} & 53.01 & \mathrm{H} & 7.33 & \mathrm{~N} & 0.87 \\ & \text { Found } & \mathrm{C} & 52.70 & \mathrm{H} & 7.25 & \mathrm{~N} & 0.86\end{array}$

RAMEB complex of $4 a(5 \mathrm{C})$ :

${ }^{1} \mathrm{H}$ NMR $\left(200 \mathrm{MHz}, \mathrm{D}_{2} \mathrm{O} /\right.$ methanol- $\left.d_{4}, 5: 2 \mathrm{v} / \mathrm{v}\right): \delta=1.62\left(\mathrm{~s}, 3 \mathrm{H}, \mathrm{CH}_{2}=\mathrm{C}\left(\mathrm{CH}_{3}\right)\right), 2.04(\mathrm{~s}$, $3 \mathrm{H}, \mathrm{C}(\mathrm{O}) \mathrm{CH}_{3}$ ), $2.81-3.13$ (m overlapped with methanol, $\mathrm{CH}_{2}$ ), 3.13 (s, RAMEB$\left.\mathrm{OCH}_{3}(6), \mathrm{H} 2\right), 3.39\left(\mathrm{RAMEB}-\mathrm{OCH}_{3}(2), \mathrm{H} 4\right), 3.40$ (RAMEB-H6, H5), 3.49 (s overlapped with RAMEB $\mathrm{H} 3$ and $\left.\mathrm{H} 5, \mathrm{OCH}_{3}\right), 3.56$ (RAMEB-H3), $4.41-4.49(2 \mathrm{~d}, 1 \mathrm{H}, \mathrm{CH})$, 4.73, $4.92\left(2 \mathrm{~s}\right.$, RAMEB-H1), $5.21\left(\mathrm{~s}, 1 \mathrm{H}, \mathrm{CH}_{2}=\mathrm{C}\right), 5.37\left(\mathrm{~s}, 1 \mathrm{H}, \mathrm{CH}_{2}=\mathrm{C}\right), 6.78\left(\mathrm{~d},{ }^{3} \mathrm{~J}=\right.$ $\left.8.30 \mathrm{~Hz}, 2 \mathrm{H}, \mathrm{ArH}_{\text {ortho }}\right), 7.06$ (d, J = $8.30 \mathrm{~Hz}, 2 \mathrm{H}, \mathrm{ArH}_{\text {meta }}$ ).

IR (diamond): $v / \mathrm{cm}^{-1}=3405$ (RAMEB-OH), 2925, 2838 (RAMEB-alkyl), $1741 \mathrm{br}$ $(\mathrm{C}=\mathrm{O}), 1663$ (amide I), $1621 \mathrm{br}(-\mathrm{C}=\mathrm{C}-), 1516$ (amide II), 1449, $1364\left(\mathrm{RAMEB}-\mathrm{CH}_{2}\right.$, $\left.-\mathrm{CH}_{3}\right), 1199$ (C-O-C), 1154, 1083, 1036, 965 (RAMEB -C-O-C-, -C-O-), 858 (1,4disubstituted benzenes), further intensive signals: 1658, 1621, 758.

\section{RAMEB complex of $\mathbf{4 b}(\mathbf{5 d})$ :}

${ }^{1} \mathrm{H}$ NMR $\left(200 \mathrm{MHz}, \mathrm{D}_{2} \mathrm{O} /\right.$ methanol- $\left.d_{4}, 5: 2 \mathrm{v} / \mathrm{v}\right): \delta=0.95\left(\mathrm{t},{ }^{3} \mathrm{~J}=7.16 \mathrm{~Hz}, 3 \mathrm{H}, \mathrm{CH}_{2} \mathrm{CH}_{3}\right)$, $1.64\left(\mathrm{~s}, 3 \mathrm{H}, \mathrm{CH}_{2}=\mathrm{C}\left(\mathrm{CH}_{3}\right)\right), 2.04\left(\mathrm{~s}, 3 \mathrm{H}, \mathrm{C}(\mathrm{O}) \mathrm{CH}_{3}\right), 2.75-3.77\left(\mathrm{~m}, \mathrm{RAMEB}-\mathrm{OCH}_{3}(6)\right.$, $\mathrm{OCH}_{3}(2), \mathrm{H} 2, \mathrm{H} 4, \mathrm{H} 6, \mathrm{H} 5, \mathrm{H} 3, \mathrm{CH}_{2}$, methanol), $3.92\left(\mathrm{q},{ }^{3} \mathrm{~J}=7.15 \mathrm{~Hz}, 2 \mathrm{H}, \mathrm{OCH}_{2} \mathrm{CH}_{3}\right.$ ), $4.42(\mathrm{t}, 1 \mathrm{H}, \mathrm{CH}), 4.74,4.94(2 \mathrm{~s}, \mathrm{RAMEB}-\mathrm{H} 1), 5.22\left(\mathrm{~s}, 1 \mathrm{H}, \mathrm{CH}_{2}=\mathrm{C}\right), 5.38(\mathrm{~s}, 1 \mathrm{H}$, $\left.\mathrm{CH}_{2}=\mathrm{C}\right), 6.80\left(\mathrm{~d},{ }^{3} \mathrm{~J}=8.30 \mathrm{~Hz}, 2 \mathrm{H}, \mathrm{ArH}_{\text {ortho }}\right), 7.08\left(\mathrm{~d}, \mathrm{~J}=8.30 \mathrm{~Hz}, 2 \mathrm{H}, \mathrm{ArH}_{\text {meta }}\right.$ ).

IR (diamond): $v / \mathrm{cm}^{-1}=3423$ (RAMEB-OH), 2931, 2837 (RAMEB-alkyl), 1762, 1739 $(\mathrm{C}=\mathrm{O}), 1666$ (amide I), 1627 (-C=C-), 1508 (amide II), 1452, 1407, 1370 (RAMEB$\mathrm{CH}_{2},-\mathrm{CH}_{3}$ ), 1198 (-C-O-C-), 1159, 1090, 1043, 968 (RAMEB -C-O-C-, -C-O-), 859 (1,4-disubstituted aromatic ring), further intensive signals: 1332, 1330, 914.

\section{Homopolymerization}

I) Polymerization of cyclodextrin-complexed monomers $5 \mathrm{a}-\mathrm{d}$ in water at $60^{\circ} \mathrm{C}$

A solution of $1.90 \mathrm{mmol}$ of complexed monomers $\mathbf{5 a - d}$ and $0.10 \mathrm{mmol}$ of $\mathrm{K}_{2} \mathrm{~S}_{2} \mathrm{O}_{8}$ and $0.10 \mathrm{mmol} \mathrm{Na} \mathrm{S}_{2} \mathrm{O}_{5}$ in $15 \mathrm{ml}$ water was stirred for $24 \mathrm{~h}$ at $60^{\circ} \mathrm{C}$. The precipitated 
polymer was filtered off, washed with water, dissolved in a small amount of THF and added dropwise to an ether solution. The polymers were collected by filtration and dried in vacuum.

Yield: 6a (70\%), 6b (90\%), 6c (57\%), 6d (70\%).

6a: $\quad$ GPC (UV): $M_{\mathrm{n}}=22338, M_{\mathrm{w}}=106260 \mathrm{~g} / \mathrm{mol}, M_{\mathrm{w}} / M_{\mathrm{n}}=4.8$.

GPC (RI): $M_{\mathrm{n}}=22817, M_{\mathrm{w}}=127370, M_{\mathrm{w}} / M_{\mathrm{n}}=5.6$.

6b: GPC (UV): $M_{\mathrm{n}}=23806, M_{\mathrm{w}}=95645, M_{\mathrm{w}} / M_{\mathrm{n}}=4.0$.

GPC (RI): $M_{\mathrm{n}}=25447, M_{\mathrm{w}}=98839, M_{\mathrm{w}} / M_{\mathrm{n}}=3.9$.

6c: $\quad$ GPC (UV): $M_{\mathrm{n}}=17200, M_{\mathrm{w}}=54043, M_{\mathrm{w}} / M_{\mathrm{n}}=3.1$.

GPC (IR): $M_{\mathrm{n}}=16148, M_{\mathrm{w}}=64441, M_{\mathrm{w}} / M_{\mathrm{n}}=4.0$.

6d: GPC (UV): $M_{\mathrm{n}}=15518, M_{\mathrm{w}}=64092, M_{\mathrm{w}} / M_{\mathrm{n}}=4.1$.

GPC (RI): $M_{\mathrm{n}}=23836, M_{\mathrm{w}}=78800, M_{\mathrm{w}} / M_{\mathrm{n}}=3.3$.

II) Polymerization of the uncomplexed monomers $\mathbf{3 a}, \mathbf{3 b}, \mathbf{4 a}$ and $\mathbf{4 b}$ in water

$0.76 \mathrm{mmol}$ of monomer was suspended in $4 \mathrm{ml}$ of freshly boiled distillated water. The suspension was flushed with nitrogen and polymerized with $5 \mathrm{~mol}-\%$ of the initiator system $\mathrm{K}_{2} \mathrm{~S}_{2} \mathrm{O}_{8} / \mathrm{Na}_{2} \mathrm{~S}_{2} \mathrm{O}_{5}$ at room temperature $\left(21-22^{\circ} \mathrm{C}\right)$. After $24 \mathrm{~h}$, precipitated polymers were filtered off, washed with water and dried in vacuum.

Yield: 6a (63\%), 6b (77\%), 6c (50\%), 6d (80\%).

6a: $\quad$ GPC (UV): $M_{\mathrm{n}}=14930, M_{\mathrm{w}}=39490, M_{\mathrm{w}} / M_{\mathrm{n}}=2.6$.

GPC (RI): $M_{\mathrm{n}}=14830, M_{\mathrm{w}}=42250, M_{\mathrm{w}} / M_{\mathrm{n}}=2.9$.

6b: $\quad$ GPC (UV): $M_{\mathrm{n}}=19150, M_{\mathrm{w}}=59500, M_{\mathrm{w}} / M_{\mathrm{n}}=3.1$.

GPC (RI): $M_{\mathrm{n}}=19990, M_{\mathrm{w}}=63650, M_{\mathrm{w}} / M_{\mathrm{n}}=3.2$.

6c: GPC (UV): $M_{\mathrm{n}}=8170, M_{\mathrm{w}}=17050, M_{\mathrm{w}} / M_{\mathrm{n}}=2.1$.

GPC (IR): $M_{\mathrm{n}}=8610, M_{\mathrm{w}}=18112, M_{\mathrm{w}} / M_{\mathrm{n}}=2.1$.

6d: GPC (UV): $M_{\mathrm{n}}=11980, M_{\mathrm{w}}=25490, M_{\mathrm{w}} / M_{\mathrm{n}}=2.1$.

GPC (RI): $M_{\mathrm{n}}=13425, M_{\mathrm{w}}=28763, M_{\mathrm{w}} / M_{\mathrm{n}}=2.1$.

III) Polymerization of uncomplexed monomers $\mathbf{3 a}, \mathbf{3 b}, \mathbf{4 a}$ and $\mathbf{4 b}$ in dimethyl formamide

$1.65 \mathrm{mmol}$ of the monomers were dissolved in $4 \mathrm{ml}$ of DMF. The solution was flushed with nitrogen and polymerized with $2 \mathrm{~mol}-\%$ of AIBN at $70^{\circ} \mathrm{C}$. After $24 \mathrm{~h}$, the cold solutions were poured into diethyl ether and the precipitated polymers were isolated by filtration, washed with diethyl ether and dried in vacuum. The polymeric powder was finely dispersed in solution and therefore it was difficult to filter off completely.

Yield: 6a (15\%), 6b (70\%), 6c (30\%), 6d (20\%).

6a: GPC (UV): $M_{\mathrm{n}}=7215, M_{\mathrm{w}}=15993, M_{\mathrm{w}} / M_{\mathrm{n}}=2.2$.

GPC (RI): $M_{\mathrm{n}}=7354, M_{\mathrm{w}}=16693, M_{\mathrm{w}} / M_{\mathrm{n}}=2.3$.

6b: $\quad$ GPC (UV): $M_{\mathrm{n}}=7451, M_{\mathrm{w}}=19211, M_{\mathrm{w}} / M_{\mathrm{n}}=2.6$.

GPC (RI): $M_{\mathrm{n}}=7549, M_{\mathrm{w}}=19530, M_{\mathrm{w}} / M_{\mathrm{n}}=2.6$.

GPC (chiralizer): $M_{\mathrm{n}}=3762, M_{\mathrm{w}}=24114, M_{\mathrm{w}} / M_{\mathrm{n}}=6.4$.

6c: GPC (UV): $M_{\mathrm{n}}=5679, M_{\mathrm{w}}=17899, M_{\mathrm{w}} / M_{\mathrm{n}}=3.1$.

GPC (IR): $M_{\mathrm{n}}=6964, M_{\mathrm{w}}=19595, M_{\mathrm{w}} / M_{\mathrm{n}}=2.8$. 
6d: GPC (UV): $M_{\mathrm{n}}=6938, M_{\mathrm{w}}=16242, M_{\mathrm{w}} / M_{\mathrm{n}}=2.3$.

GPC (RI): $M_{\mathrm{n}}=6943, M_{\mathrm{w}}=14514, M_{\mathrm{w}} / M_{\mathrm{n}}=2.1$.

IV) Polymerization of uncomplexed monomers $\mathbf{3 a}, \mathbf{3 b}, \mathbf{4 a}$ and $\mathbf{4 b}$ in tetrahydrofuran

A solution of $1.90 \mathrm{mmol}$ of monomers $\mathbf{3 a}, \mathbf{3 b}, \mathbf{4 a}$ and $\mathbf{4 b}$ and $0.06 \mathrm{mmol}$ of AIBN in 15 $\mathrm{ml}$ THF was stirred for $24 \mathrm{~h}$ at $70^{\circ} \mathrm{C}$ under nitrogen. After the amount of solvent was reduced to $5 \mathrm{ml}$, the solution was dropped into petroleum ether. The precipitated polymers were filtered off, washed with petroleum ether and dried in vacuum.

Yield: 6a (52\%), 6b (78\%), 6c (60\%), 6d (77\%).

6a: GPC (UV): $M_{\mathrm{n}}=6182, M_{\mathrm{w}}=13069, M_{\mathrm{w}} / M_{\mathrm{n}}=2.1$.

GPC (RI): $M_{\mathrm{n}}=6444, M_{\mathrm{w}}=17145, M_{\mathrm{w}} / M_{\mathrm{n}}=2.7$.

6b: $\quad$ GPC (UV): $M_{\mathrm{n}}=6254, M_{\mathrm{w}}=17712, M_{\mathrm{w}} / M_{\mathrm{n}}=2.8$.

GPC (RI): $M_{\mathrm{n}}=6456, M_{\mathrm{w}}=18500, M_{\mathrm{w}} / M_{\mathrm{n}}=2.9$.

6c: $\quad$ GPC (UV): $M_{\mathrm{n}}=4043, M_{\mathrm{w}}=9830, M_{\mathrm{w}} / M_{\mathrm{n}}=2.4$.

GPC (IR): $M_{\mathrm{n}}=4354, M_{\mathrm{w}}=9747, M_{\mathrm{w}} / M_{\mathrm{n}}=2.2$.

6d: $\quad$ GPC (UV): $M_{\mathrm{n}}=5381, M_{\mathrm{w}}=9756, M_{\mathrm{w}} / M_{\mathrm{n}}=1.8$.

GPC (RI): $M_{\mathrm{n}}=5590, M_{\mathrm{w}}=9048, M_{\mathrm{w}} / M_{\mathrm{n}}=1.6$.

\section{${ }^{1} \mathrm{H}$ NMR analysis of polymers $6 \mathbf{a}-\boldsymbol{d}$}

6a: $\left(300 \mathrm{MHz}, \mathrm{DMSO}-d_{6}\right) \delta=0.78\left(3 \mathrm{H}, \mathrm{CH}_{3}\right), 1.48\left(2 \mathrm{H}, \mathrm{CH}_{2}\right), 2.87\left(\mathrm{~s}, 2 \mathrm{H}, \mathrm{CH}_{2}\right), 3.43$ $\left(3 \mathrm{H}, \mathrm{OCH}_{3}\right), 4.33(\mathrm{~s}, 1 \mathrm{H}, \mathrm{CH}), 6.65(2 \mathrm{H}$, aromatic $\mathrm{H}), 6.94(2 \mathrm{H}$, aromatic $\mathrm{H}), 7.10(1 \mathrm{H}$, $\mathrm{OH}), 9.25(1 \mathrm{H}, \mathrm{NH})$.

6b: $\left(300 \mathrm{MHz}, \mathrm{DMSO}-d_{6}\right) \delta=0.60-1.30$ (br, aliphatic $\left.\mathrm{H}\right), 1.36-2.00$ (br, aliphatic $\mathrm{H}), 2.53$ - 3.15 (br, aliphatic $\mathrm{H}), 3.6$ - $4.08\left(\mathrm{br}, \mathrm{OCH}_{2}\right), 4.20$ - $4.40(\mathrm{br}, \mathrm{CH}), 6.35$ - 6.66 (aromatic $\mathrm{H}), 6.95$ - 7.38 (br, aromatic $\mathrm{H}, \mathrm{OH}), 9.23(\mathrm{NH})$.

6c: $\left(300 \mathrm{MHz}, \mathrm{DMSO}-d_{6}\right) \delta=0.77-2.28$ (br m, aliphatic H), $2.86-3.17$ (br, aliphatic $\mathrm{H}), 3.43\left(\mathrm{br}, \mathrm{OCH}_{3}\right), 4.35$ - $4.68(\mathrm{br}, \mathrm{CH}), 6.55$ - 6.66 (br m, aromatic H), 6.94 - 6.98 (br m, aromatic $\mathrm{H}), 9.22$ (br, $\mathrm{NH})$.

6d: $\left(300 \mathrm{MHz}, \mathrm{CDCl}_{3}\right) \delta=0.2-2.2$ (br m, aliphatic H), $2.72-3.25$ (br, $\left.\mathrm{CH}_{2}\right), 3.77$ $4.23\left(\mathrm{br}, \mathrm{OCH}_{2}\right), 4.69$ - $4.95(\mathrm{br}, \mathrm{CH}), 6.99$ (br, aromatic $\left.\mathrm{H}\right), 7.20$ (br, aromatic $\left.\mathrm{H}\right)$, $7.33-7.94(\mathrm{br}, \mathrm{NH})$.

\section{IR analysis of polymers 6a-d (polymerization in $\mathrm{H}_{2} \mathrm{O}$ without cyclodextrin)}

6a: IR (diamond) v/cm ${ }^{-1}=3360$ br $(\mathrm{NH}, \mathrm{OH}), 3013,2954$ br (alkyl), $1726(\mathrm{C}=\mathrm{O}), 1639$ (amide I), 1514 (amide II), 1443, $1370\left(-\mathrm{CH}_{2},-\mathrm{CH}_{3}\right), 1222$ (-C-O-), further intensive signals: $1614,1596,1201,1108,1021,828$.

6b: IR (diamond) $v / \mathrm{cm}^{-1}=3357 \mathrm{br}(\mathrm{NH}, \mathrm{OH}), 2982,2937 \mathrm{br}$ (alkyl), $1723(\mathrm{C}=\mathrm{O}), 1640$ (amide I), 1514 (amide II), 1446, $1374\left(-\mathrm{CH}_{2},-\mathrm{CH}_{3}\right), 828$ (1,4-disubstituted aromatic ring), further intensive signals: 1614, 1596, 1349, 1217, 1198, 1108 and 1022.

6c: IR (diamond) $v / \mathrm{cm}^{-1}=3358$ br $(\mathrm{NH}), 3025$ (aryl), 2954 br (alkyl), $1726(\mathrm{C}=\mathrm{O})$, 1637 (amide I), 1514 (amide II), 1445, $1372\left(-\mathrm{CH}_{2},-\mathrm{CH}_{3}\right), 827$ (1,4-disubstituted 
aromatic ring), further intensive signals: 1614, 1596, 1351, 1219, 1198, 1108 and 1020.

6d: IR (diamond) v/cm ${ }^{-1}=3380 \mathrm{br}(\mathrm{NH}), 2985 \mathrm{br}$ (alkyl), 1756, $1728(\mathrm{C}=\mathrm{O}), 1659$ (amide I), 1508 (amide II), 1445, $1370\left(-\mathrm{CH}_{2},-\mathrm{CH}_{3}\right), 857$ (1,4-disubstituted aromatic ring), further intensive signals: 1192, 1167, 1109, 1018, 913.

Acknowledgement: We thank Dr. D. Schollmeyer and Dipl.-Chem. H. Kolshorn from the University of Mainz, Germany, for X-ray analyses and 2D NMR experiments.

[1] Szejtli, J.; Osa T.; "Cyclodextrins", in Comprehensive Supramolecular Chemistry, vol. 3, Pergamon Press, Oxford 1996.

[2] Szejtli, J.; “Cyclodextrin Technology”, Kluwer Academic Publisher, Dordrecht 1998.

[3] Okada, M.; Kamachi, M.; Harada; A.; Macromolecules 1999, 32, 7202.

[4] Wenz, G.; Angew. Chem. 1994, 106, 851.

[5] Harada, A.; Acta Polym. 1998, 49, 3.

[6] Hermann, W.; Keller, B.; Wenz, G.; Macromolecules 1997, 28, 4966.

[7] Born, M.; Ritter, H.; Angew. Chem. 1994, 106, 851.

[8] Jeromin, J.; Noll, O.; Ritter, H.; Macromol. Chem. Phys. 1998, 199, 2641.

[9] Jeromin, J.; Ritter, H.; Macromol. Rapid. Commun. 1998, 19, 377.

[10] Jeromin, J.; Ritter, H.; Macromolecules 1999, 32, 5236.

[11] Glöckner, P.; Ritter, H.; Macromol. Rapid Commun. 1999, 20, 602.

[12] Fischer, M.; Ritter, H.; Macromol. Rapid Commun. 2000, 21, 142.

[13] Storsberg, J.; Hartenstein, M.; Müller, A. H. E.; Ritter, H.; Macromol. Rapid Commun. 2000, 21, 1342.

[14] Storsberg, J.; Ritter, H.; Macromol. Rapid Commun. 2000, 21, 236.

[15] Casper, P.; Glökner, P.; Ritter, H.; Macromolecules 2000, 33, 4361.

[16] Alupei, V.; Ritter, H.; Macromol. Rapid Commun. 2001, 22, 1349.

[17] Bernhardt, S.; Glöckner, P.; Theis, A.; Ritter, H.; Macromolecules 2001, 34, 1647

[18] Storsberg, J.; Glöckner, P.; Eigner, M.; Schnöller, U.; Ritter, H.; Voit, B.; Nuyken, O.; Designed Monomers and Polymers 2001, 4, 9.

[19] Storsberg, J.; Ritter, H.; Macromol. Chem. Phys. 2002, 203, 812.

[20] Blaschke, G.; J. Liq. Chromatogr. 1986, 9, 341.

[21] Okamoto, Y.; Angew. Chem. 1998, 110, 1072.

[22] Pirkle, W.; Chem. Rev. 1989, 89, 347. 